Disclosure No significant relationships.

\section{P634 SURVEILLANCE FOR DISSEMINATED GONOCOCCAL INFECTIONS, ACTIVE BACTERIAL CORE SURVEILLANCE (ABCS) - UNITED STATES, 2015-2018}

\begin{abstract}
${ }^{1}$ Emily Weston*, ${ }^{2}$ Mirasol Apostol, ${ }^{3}$ Ashley Moore, ${ }^{4}$ Amy Tunali, ${ }^{4}$ Monica Farley, ${ }^{5}$ Samera Sharpe, ${ }^{5}$ Audrey Jeanine Mclean, ${ }^{6}$ Kimberly Workowski, ${ }^{5}$ Elizabeth Torrone, ${ }^{5}$ Hillard Weinstock. ' US Centers for Disease Control and Prevention, Division of Sexually Transmitted Diseases, Atlanta, USA; ${ }^{2}$ California Emerging Infections Program, Oakland, USA; ${ }^{3}$ Georgia Department of Public Health, Atlanta, USA; ${ }^{4}$ Georgia Emerging Infections Program, Emory University School of Medicine, Atlanta, USA; ${ }^{5}$ US Centers for Disease Control and Prevention, Division of STD Prevention, Atlanta, USA; ${ }^{6}$ US Centers for Disease Control and Prevention, Division of STD Prevention, Emory University Department of Medicine, Atlanta, USA
\end{abstract}

10.1136/sextrans-2019-sti.702

Background Disseminated gonococcal infections (DGI) are uncommon; occurring in an estimated $0.5-3 \%$ of Neisseria gonorrhoeae (GC) cases. DGI surveillance is limited and case reports are often analyzed retrospectively or in case clusters. We describe the population-level burden of laboratory culture confirmed DGI using an established surveillance infrastructure, the Active Bacterial Core surveillance (ABCs) system of CDC's Emerging Infections Program.

Methods During 2017-2018, prospective surveillance was conducted among residents in three ABCs areas (3-counties in the Bay Area in California (CA), the 20-county Atlanta metropolitan area in Georgia [GA-MSA], and Georgia outside of the 20-county metropolitan area [GA-DPH]); retrospective surveillance was conducted during 2015-2016 in CA and GA-MSA. A DGI case was defined as isolation of GC from a normally sterile site; a case report form was completed for each case. Isolates collected during prospective surveillance underwent antimicrobial susceptibility testing (AST).

Results During 2015-2018, 53 DGI cases were identified (12 in CA, 6 in GA-DPH, and 41 in GA-MSA) for an overall rate of 0.11 cases per 100,000 population $(0.08$ per 100,000 in CA, 0.06 in GA-DPH, 0.16 in GA-MSA). DGI cases accounted for $0.06 \%$ of all reported cases of GC in the three surveillance areas. Most DGI cases were male $(60 \%)$, aged $15-29$ years $(34 \%)$ or $\geq 45$ years $(34 \%)$, and were Black, non-Hispanic (58\%). Clinical presentation was bacteremia $(23 \%)$ or monoarticular septic arthritis $(26 \%)$; $11 \%$ were immunocompromised. Of the 37 cases identified during 2017-2018, 18 viable isolates had AST completed. All were susceptible to Azithromycin, Ceftriaxone, and Cefixime; 6 (33\%) were Penicillin and/or Ciprofloxacin resistant.

Conclusion DGI is an infrequent complication of GC. The $\mathrm{ABCs}$ infrastructure is a viable platform for DGI surveillance. As GC can quickly develop antimicrobial resistance, continued surveillance, including monitoring trends in antimicrobial susceptibility of DGI isolates and molecular epidemiology, could help inform DGI treatment recommendations.

Disclosure No significant relationships.

\section{P635 THE ENHANCED GONOCOCCAL ANTIMICROBIAL SURVEILLANCE PROGRAM (EGASP) IN THE PHILIPPINES, 2018}

${ }^{1}$ Emily Weston*, ${ }^{2}$ Mark Angelo Amoroso, ${ }^{2}$ Noel Palapayon, ${ }^{2}$ Genesis Samonte, ${ }^{3}$ Celia Carlos, ${ }^{1}$ Cau Pham, ${ }^{4}$ Teodora Wi. ${ }^{1}$ US Centers for Disease Control and Prevention, Division of STD Prevention, Atlanta, USA; ${ }^{2}$ Philippines Department of Health, National HIVI AIDS and STI Surveillance and Strategic Information Unit, Epidemiology Bureau, Manila, Philippines; ${ }^{3}$ Philippines Department of Health, Research Institute of Tropical Medicine, Manila, Philippines; ${ }^{4}$ World Health Organization, Department of Reproductive Health and Research, Geneva, Switzerland

\subsection{6/sextrans-2019-sti.703}

Background In July 2018, the Philippines Department of Health, the Centers for Disease Control and Prevention and World Health Organization implemented the Enhanced Gonococcal Antimicrobial Surveillance Program (EGASP). The Philippines is the second country to implement EGASP to monitor antimicrobial susceptibility trends of Neisseria gonorrhoeae (GC).

Methods Men with urethral discharge were enrolled at 4 participating sentinel clinics in Metro Manila. Symptomatic men had demographic and clinical data collected; two urethral swabs were collected for gram stain and culture. All positive cultures had antimicrobial susceptibility testing (AST) performed by 2 participating reference laboratories to determine minimum inhibitory concentrations for Cefixime, Ceftriaxone, Azithromycin, and Gentamicin using Etest.

Results From July - September 2018, 92 specimens were collected from symptomatic men; 61 (66\%) were culture confirmed and all GC isolates had AST. Among 60 men with at least one GC infection, 32 (53\%) were classified as men who have sex with women only, $15(25 \%)$ as men who have sex with men and $13(22 \%)$ were men who have sex with men and women. The median age of men was 24 years (range 15 -52 years), $12(20 \%)$ had antibiotic use in the last 2 weeks, 4 (7\%) had travel in the last 30 days, and 43 (72\%) received combination therapy of Ceftriaxone $(250 \mathrm{mg}, 500 \mathrm{mg}$ or $1 \mathrm{~g}$ ) or Cefixime $400 \mathrm{mg}$ PLUS Doxycycline $100 \mathrm{mg}$ or Azithromycin 1 g. Only $6(10 \%)$ did not have treatment documented. No isolates had elevated MICs to the antimicrobials tested. Conclusion We report on the first months of data from EGASP Philippines demonstrating the feasibility of collecting standardized and systematic epidemiological and laboratory data. All isolates were susceptible to all tested antimicrobials. Continued surveillance is critical to monitor for emerging resistance and to inform local and regional treatment recommendations.

Disclosure No significant relationships. 